\title{
GABOR FRAMES AND OPERATOR ALGEBRAS
}

\author{
J-P. Gabardo ${ }^{a}$, Deguang $\operatorname{Han}^{*}$, David R. Larson ${ }^{b}$ \\ ${ }^{a}$ Dept of Math \& Statistics, McMaster University, Hamilton, Canada \\ ${ }^{b}$ Dept. of Mathematics, Texas A\&M University, College Station, TX
}

\begin{abstract}
We discuss three applications of operator algebra techniques in Gabor analysis: the parametrizations of Gabor frames, the incompleteness property, and the unique Gabor dual problem for subspace Gabor frames.
\end{abstract}

Keywords: Gabor frame, operator algebra, dual frame, density theorem. Appeared in Proc. SPIE., 4119 (2000), $337-345$.

\section{INTRODUCTION}

In 1946, D. Gabor in his "Theory of Communication" [16] formulated a fundamental approach to signal decomposition in terms of elementary signals. His approach has become the paradigm for the spectral analysis associated with timefrequency methods, and more uses are being found for the theory such as optics, filterbanks, signal detection and many other applications. Traditionally Gabor and wavelet analysis was studied by using classical Fourier analysis methods. But in recent years more abstract tools (operator algebra, abstract harmonic analysis and Group-representations, etc) have been introduced. Gabor analysis actually has it roots in von Neumann algebra theory which can be traced back to von Neumann's work [29] in the 30's about the von Neumann lattice, and the relatively recent work [26] in the 80 's by Rieffel about the incompleteness property for Gabor families. In the past few years the authors of this article and the co-authors have been interested in working on some basic problems on wavelets and Gabor frames from a functional analysis point of view, and some of the results we obtained were reported in [5], [8], [13], [14], [15], [18] [19], [20], [21], [24], [25] etc. The purpose of this paper is to discuss a few applications of operator algebra and operator theory in Gabor analysis. For other recent developments on Gabor analysis, we refer to the excellent survey papers in the book [12] edited by H.G. Feichtinger and T. Strohmer and the survey paper [2] by Pete Casazza (see also $[1,[3],[4]$ etc).

Let $\mathcal{L}$ and $\mathcal{K}$ be two full-rank lattices in $R^{d}$, and let $g(x) \in L^{2}\left(R^{d}\right)$. The Gabor family associated with $\mathcal{L}, \mathcal{K}$ and $g$ is the collection:

$$
\mathbf{G}(\mathcal{L}, \mathcal{K}, g):=\left\{e^{2 \pi i<\ell, x>} g(x-\kappa) \mid \ell \in \mathcal{L}, \kappa \in \mathcal{K}\right\}
$$

In signal processing, it is often required that the Gabor family is either an orthonormal basis or a frame of $L^{2}\left(R^{d}\right)$. Recall that a family of vectors $\left\{f_{j}\right\}$ in a separable Hilbert space $H$ is a frame if there exist constants $C_{1}, C_{2}>0$ such that

$$
C_{1}\|f\|_{2}^{2} \leq \sum_{j}\left|<f, f_{j}>\right|^{2} \leq C_{2}\|f\|_{2}^{2}
$$

for all $f \in H$. If $C_{1}=C_{2}=1$ we say that $\left\{f_{j}\right\}$ is a normalized tight frame, and $\left\{f_{j}\right\}$ is called a Bessel sequence if at least the upper frame bound in is satisfied.

For any $(s, t) \in R^{d \times d}$, the translation and modulation unitary operators are defined by:

$$
\mathcal{T}_{t} f(x)=f(x-t)
$$

and

$$
\left(\mathcal{E}_{s} f\right)(x)=e^{2 \pi i<s, x>} f(x)
$$

${ }^{*}$ Correspondence: Email: hand@icarus.math.mcmaster.ca; Telephone: (905)525-9140 ext. 27108 
for all $f \in L^{2}\left(R^{d}\right)$. Write $\mathcal{U}_{\mathcal{L}, \mathcal{K}}=\left\{\mathcal{E}_{\ell} \mathcal{T}_{\kappa}: \ell \in \mathcal{L}, \kappa \in \mathcal{K}\right\}$. Then it is easy to check by the definition that the group generated by $\mathcal{U}_{\mathcal{L}, \mathcal{K}}$ is contained in $T \mathcal{U}_{\mathcal{L}, \mathcal{K}}$, where $T$ denotes the unit circle. We will call $\mathcal{U}_{\mathcal{L} . \mathcal{K}}$ a Gabor unitary system. More generally, a unitary system (see [8]) is a countable set of unitary operators $\mathcal{U}$ acting on a separable Hilbert space $H$ which contains the identity operator. Denoting by $\operatorname{group}(\mathcal{U})$ the group generated by $U$ and letting $T=\{\lambda \in C:|\lambda|=1\}$, we call $\mathcal{U}$ a group-like unitary system if $\mathcal{U}$ has the property that

$$
\operatorname{group}(\mathcal{U}) \subset T \mathcal{U}:=\{\lambda U: \lambda \in T, U \in \mathcal{U}\}
$$

and if different $U$ and $V$ in $\mathcal{U}$ are always linearly independent. Typical examples include group unitary systems, Gabor-type unitary systems (cf [18], [20]), the Gabor unitary systems and their restriction to any of their invariant subspaces.

For a unitary system $\mathcal{U}$, a vector $\xi \in H$ is called a frame vector (resp. normalized tight frame vector or Bessel) for $\mathcal{U}$ if $\{U \xi\}_{U \in \mathcal{U}}$ is a frame (resp. normalized tight frame or Bessel family) for $[\mathcal{U} \xi]$, the closure of the linear span of $\{U \xi\}_{U \in \mathcal{U}}$. When $[\mathcal{U} \xi]=H$, then the frame vector is said to be complete. In case that $\{U \xi: U \in \mathcal{U}\}$ is an orthonormal basis (resp. Riesz basis) for $H, \xi$ is said to be a complete wandering vector (resp. complete Riesz vector) for $\mathcal{U}$. A Bessel vector $\xi$ is called a dual frame vector of $\eta$ if $\mathcal{U} \xi$ is a dual for $\mathcal{U} \eta$, i.e.

$$
x=\sum_{U \in \mathcal{U}}<x, U \xi>U \eta
$$

holds for all $x \in H$, where the convergence is in the norm topology of $H$. More generally, if $\left\{x_{i}\right\}$ and $\left\{y_{i}\right\}$ are two frames for a Hilbert space $H$ such that

$$
x=\sum_{i}<x, y_{i}>x_{i}
$$

holds for all $x \in H$, then $\left\{y_{i}\right\}$ will be called a dual frame of $\left\{x_{i}\right\}$. It is well known that a frame has a unique dual if and only if it is a Riesz basis.

Let $\mathcal{U}$ be a group-like unitary system. We will denote by $l^{2}(\mathcal{U})$ the Hilbert space of square-summable sequences indexed by $\mathcal{U}$ and by $B_{\mathcal{U}}$ the set of all Bessel vectors for $\mathcal{U}$. It is clear that $B_{\mathcal{U}}$ is invariant under the commutant $\mathcal{U}^{\prime}=\{T \in B(H): T U=U T, U \in \mathcal{U}\}$, i.e., $T x \in B_{\mathcal{U}}$ if $x \in B_{\mathcal{U}}$ and $T \in \mathcal{U}^{\prime}$.

Given $x \in H$, the analysis operator $T_{x}$ is defined by

$$
T_{x} y=\sum_{U \in \mathcal{U}}<y, U x>\chi_{U}
$$

for all $y \in H$ such that $\{\langle y, U x\rangle\}_{U \in \mathcal{U}}$ is in $l^{2}(\mathcal{U})$, where $\chi_{U}$ denotes the element in $l^{2}(\mathcal{U})$ which takes value 1 at $U$ and 0 everywhere else. Clearly, $x \in B_{\mathcal{U}}$ if and only if $T_{x}$ is a bounded linear operator from $H$ into $l^{2}(\mathcal{U})$. In general, if $B_{\mathcal{U}}$ is dense in $H$, then $T_{x}$ would be a densely defined and closed operator for each $x \in H$. Let $\eta$ be a complete frame vector for a group-like unitary system $\mathcal{U}$ acting on $H$. The $S=T_{\eta}^{*} T_{\eta}$ is an invertible positive operator on $H$. It is easy to verify by the definition of analysis operator that $S \in \mathcal{U}^{\prime}$ and $S^{-1 / 2} \eta$ is a complete normalized tight frame vector for $\mathcal{U}$. Moreover,

$$
x=\sum_{U \in \mathcal{U}}<x, U S^{-1} \eta>U \eta, \quad x \in H .
$$

Thus $S^{-1} \eta$ is a dual frame vector for $\eta$, which is called the standard dual frame vector.

A von Neumann algebra is a *-subalgebra of $B(H)$ such that it contains the identity operator $I$ and is closed in the weak operator (or strong operator ) topology. We use $w^{*}(\mathcal{U})$ to denote the von Neumann algebra generated by $\mathcal{U}$. We will also use $M$ to denote the von Neumann algebra generated by $\mathcal{U}_{\mathcal{L}, \mathcal{K}}$ for two full rank lattices in $R^{d}$.

For a given group-like unitary system $\mathcal{U}$, we investigate three questions about the parametrizations of frame vectors, the incompleteness property and the characterization of the frame vector that has a unique dual frame vector. Applying these results to Gabor systems, we are able to obtain some new results, as well as some new approach to old problems. Details about each question will be given in the the corresponding section. 


\section{PARAMETRIZATIONS OF GABOR FRAMES}

One of the basic problems in Gabor analysis is to identify those functions $g$ such that $\mathbf{G}(\mathcal{L}, \mathcal{K}, g)$ is a frame for $L^{2}\left(R^{d}\right)$. There have been some very nice and useful sufficient or necessary conditions for a function $g$ to be a generator for a Gabor frame (cf. [2]). One of the natural questions we are interested in here is the following:

Question 1: Let $\mathcal{L}$ and $\mathcal{K}$ be two full-rank lattices in $R^{d}$. how large is the set of all the functions that generate Gabor frames for $L^{2}\left(R^{d}\right)$ ?

It seems to us that, even in the one-dimensional case $(d=1, \mathcal{L}=\alpha Z, \mathcal{K}=\beta Z)$, those known sufficient conditions (for a function to generate a Gabor frame) do not provide an answer to this question except for some special cases such as the case $\alpha \beta=1$. In this special case one can use Zak transform (see definition in Section 4) to deduce the fact that the set of all the functions that generate Gabor frames (in fact Riesz bases in this case) is dense in $L^{2}\left(R^{d}\right)$. In order to answer the above question in general, we will use some operator algebra techniques to prove the following parametrization result (Theorem 2.2) for arbitrary group-like unitary systems. We start with the following special case which was observed by Dai and Larson [8] for more general unitary systems.

Proposition 2.1 Suppose that $\eta$ is a complete wandering vector for a group-like unitary system $\mathcal{U}$ on a Hilbert space $H$. Then a vector $\xi$ is a complete wandering vector for $\mathcal{U}$ if and only if there is a (unique) unitary operator $T \in \mathcal{U}^{\prime}$ such that $\xi=T \eta$. Similarly, $\xi$ is a complete Riesz vector for $\mathcal{U}$ if and only if there is a (unique) invertible operator $S$ in $\mathcal{U}^{\prime}$ such that $\xi=S \eta$.

Proof. Define $T: H \rightarrow H$ by $T U \eta=U \xi$ for all $U \in \mathcal{U}$. Then $T$ is well-defined and unitary if and only if $\mathcal{U} \xi$ is an orthonormal basis for $H$. It is also easy to check that $T \in \mathcal{U}^{\prime}$ and is unique. The second statement follows from a similar argument.

The above proposition provides us a parametrization of all the complete wandering vectors in terms of the unitary operators in the commutant $\mathcal{U}^{\prime}$ (which is a von Neumann algebra) of $\mathcal{U}$ and a fixed complete wandering vector. Moreover, the correspondence is one to one and onto. However, this is no longer true in general if we replace complete wandering vector by normalized tight frame vector (In section 4, Theorem 4.1, we will give a characterization for those normalized tight frames vectors that have the property described in Proposition 2.1). But the following result can be regarded as a "substitute" ([15], [20]).

Theorem 2.2 Let $\eta$ be a normalized tight frame vector for a group-like unitary system $\mathcal{U}$ on a Hilbert space $H$ and let $\xi \in H$. Then we have

(i) $\xi$ is a complete frame vector for $\mathcal{U}$ if and only if there is an invertible operator $T \in w^{*}(\mathcal{U})$ such that $\xi=T \eta$.

(ii) $\xi$ is a complete normalized tight frame vector for $\mathcal{U}$ if and only if there is a unitary operator $T \in w^{*}(\mathcal{U})$ such that $\xi=T \eta$.

Theorem 2.2 can be regarded as a parametrization result for all the complete frame vectors by the set of all the invertible operators in the von Neumann algebra generated the the unitary system. We remark that unlike the complete wandering vector case, the correspondence is not one to one in general. Applying Theorem 2.2 to the Gabor unitary system $\mathcal{U}_{\mathcal{L}, \mathcal{K}}$, we get

Corollary 2.3 Suppose that $\mathbf{G}(\mathcal{L}, \mathcal{K}, g)$ is a normalized tight frame for $L^{2}\left(R^{d}\right)$ and $h \in L^{2}\left(R^{d}\right)$.

(i) $\mathbf{G}(\mathcal{L}, \mathcal{K}, h)$ is a frame for $L^{2}\left(R^{d}\right)$ if and only if there is an invertible operator $T \in M$ such that $h=T g$.

(ii) $\mathbf{G}(\mathcal{L}, \mathcal{K}, h)$ is a normalized tigh frame for $L^{2}\left(R^{d}\right)$ if and only if there is a unitary operator $T \in M$ such that $h=T g$. 
Let $\mathbf{G}(\mathcal{L}, \mathcal{K})$ be the set of all the functions $h$ such that $\mathbf{G}(\mathcal{L}, \mathcal{K}, h)$ is a Gabor frame for $L^{2}\left(R^{d}\right)$, and respectively, $\mathbf{N G}(\mathcal{L}, \mathcal{K})$ the set of all $h$ such that $\mathbf{G}(\mathcal{L} . K . h)$ is a normalized tight frame for $L^{2}\left(R^{d}\right)$. Let $\mathrm{v}(\mathcal{L})$ denote the volume of the foundamental domain of $\mathcal{L}$, i.e. $\mathrm{v}(\mathcal{L})=|\operatorname{det}(A)|$ for $\mathcal{L}=A Z^{d}$. Then we have

Theorem 2.4 Suppose that $\mathrm{v}(\mathcal{L}) \mathrm{v}(\mathcal{K}) \leq 1$. Then $\mathbf{G}(\mathcal{L}, \mathcal{K})$ is dense in $L^{2}\left(R^{d}\right)$. Moreover, both $\mathbf{G}(\mathcal{L}, \mathcal{K})$ and $\mathbf{N G}(\mathcal{L}, K)$ are path connected sets in the norm topology of $L^{2}\left(R^{d}\right)$.

Proof. It is a well-known fact the the set of all the invertible operators in a von Neumann algebra is a dense subset in the strong operator topology. Since $\mathrm{v}(\mathcal{L}) \mathrm{v}(\mathcal{K}) \leq 1, \mathbf{N G}(\mathcal{L}, \mathcal{K})$ is not empty (see [22], or Theorem 3.3 below). Now let us fix a normalized tight Gabor frame $\mathbf{G}(\mathcal{L}, \mathcal{K}, g)$, and let $f \in L^{2}\left(R^{d}\right)$ be an arbitrary but fixed function. For any $\varepsilon>0$, there exits $c_{\ell, \kappa} \in C,(\ell, \kappa) \in \Lambda$ such that

$$
\left\|f-\sum_{(\ell, \kappa) \in \Lambda} c_{\ell, \kappa} \mathcal{E}_{\ell} \mathcal{T}_{\kappa} g\right\|<\varepsilon / 2
$$

where $\Lambda$ is a finite subset of $\mathcal{L} \times \mathcal{K}$. Clearly $A:=\sum_{(\ell, \kappa) \in \Lambda} c_{\ell, \kappa} \mathcal{E}_{\ell} \mathcal{T}_{\kappa} \in M$. Thus, since the set of all the invertible operators in $M$ is dense in $M$ in the strong operator topology, there is an invertible operator $T \in M$ such that $\|T g-A g\|<\epsilon / 2$. By Corollary 2.3, Tg generates a Gabor frame and

$$
\|T g-f\| \leq\|T g-A g\|+\|A g-f\|<\epsilon .
$$

Therefore $\mathbf{G}(\mathcal{L}, \mathcal{K})$ is dense in $L^{2}\left(R^{d}\right)$.

The second statement follows from Corollary 2.3 and the fact that the set of all the invertible operators in $M$ (resp. the set of unitary operators) is path-connected in the operator norm topology.

We remark that Theorem 2.4 is clearly true for any group-like unitary system $\mathcal{U}$, provided that $\mathcal{U}$ has a complete frame vector. The other consequence of Theorem 2.2 (ii) is the following (which is an analogy of a similar result for the orthogonal wavelet case [25]):

Corollary 2.5 Supose that $\mathbf{G}(\mathcal{L}, \mathcal{K}, g)$ and $\mathbf{G}(\mathcal{L}, \mathcal{K}, h)$ are normalized tight frames for $L^{2}\left(R^{d}\right)$. Then $\lambda g+(1-\lambda) h$ generates a Gabor frame for $L^{2}\left(R^{d}\right)$ whenever $\lambda \neq \frac{1}{2}$.

Proof. Write $h=T g$ for some unitary operator $T$ in $M$. Then $\lambda g+(1-\lambda) h=\lambda\left(I+\frac{1-\lambda}{\lambda} T\right) g$. Note that $I+\frac{1-\lambda}{\lambda} T \in M$. Thus in order to have a Gabor frame we need this operator to be invertible(by Corollary 2.2). So the corollary follows from the fact that the spectrum of the unitary operator $T$ is contained in the unit circle.

\section{INCOMPLETENESS PROPERTY}

The other well known question in Gabor analysis is the so-called incompleteness (or density) problem for Gabor families:

Question 2: Let $\mathcal{L}$ and $\mathcal{K}$ be two full-rank lattices in $R^{d}$. Under what conditions can we find a function $g \in L^{2}\left(R^{d}\right)$ such that the Gabor family $\mathbf{G}(\mathcal{L}, \mathcal{K}, g)$ is a frame (or more generally, a complete set) for $L^{2}\left(R^{d}\right)$ ?

In the one dimensional case the answer to Question 2 is very well-known: Let $\mathcal{L}=\alpha Z$ and $\mathcal{K}=\beta Z$. Then there is a function $g \in L^{2}(R)$ such that the Gabor family $\mathbf{G}(\mathcal{L}, \mathcal{K}, g)$ is a frame (or more generally, a complete set) for $L^{2}(R)$ if and only if $|\alpha \beta| \leq 1$. In fact, if $g=\chi_{[0,|\beta|]}$, it is easily checked that $\mathbf{G}(\mathcal{L}, \mathcal{K}, g)$ is a frame when $|\alpha \beta| \leq 1$ and a Riesz basis when $|\alpha \beta|=1$. On the other hand, the density theorem tells us that the condition $|\alpha \beta| \leq 1$ is also necessary for $\mathbf{G}(\mathcal{L}, \mathcal{K}, g)$ to be a frame (or just a complete set), and that $|\alpha \beta|=1$ is necessary for $\mathbf{G}(\mathcal{L}, \mathcal{K}, g)$ to be an orthonormal basis (see [9], [10] and [26]). Analogous necessary conditions for the high dimensional case have been established (see [28], [27], [17], [6] and [23]). Let $\mathcal{L}=A Z^{d}$ and $\mathcal{K}=B Z^{d}$ where $A$ and $B$ are real $d \times d$ invertible 
matrices. The density result states that one necessarily has $|\operatorname{det}(A B)|=1$ if $\mathbf{G}(\mathcal{L}, \mathcal{K}, g)$ is an orthonormal basis, and $|\operatorname{det}(A B)| \leq 1$ if $\mathbf{G}(\mathcal{L}, \mathcal{K}, g)$ is a complete set for $L^{2}\left(R^{d}\right)$. Interestingly the converse, which is trivial in the one dimension, remained unsolved until very recently. In [22] the converse was proved by studying a seemingly unrelated problem concerning lattice tiling in $R^{d}$.

In [13] and [14], we introduced a very simple and general approach to the incompleteness property for arbitrary group-like unitary systems. In particular, we can apply our general results to the Gabor unitary systems and their restrictions to invariant subspaces to get various density results (see [14] for more details). Here is the simple idea for our approach:

Let $\mathcal{U}$ be a group-like unitary system on $H$. By definition, there exists a function $f: \operatorname{group}(\mathcal{U}) \rightarrow T$ and a mapping $\sigma: \operatorname{group}(\mathcal{U}) \rightarrow \mathcal{U}$ such that $W=f(W) \sigma(W)$ for all $W \in \operatorname{group}(\mathcal{U})$. To see that $f$ and $\sigma$ are well defined, let $W=\lambda_{1} U_{1}=\lambda_{2} U_{2}$ with $U_{1}, U_{2} \in \mathcal{U}\left(\lambda_{1}, \lambda_{2} \in T\right)$. Then $U_{1}=U_{2}$ and $\lambda_{1}=\lambda_{2}$ since $\mathcal{U}$ is an independent set. Hence both $f$ and $\sigma$ are well defined. Using this we can define the left (resp. right) regular representation as in the group case.

For each fixed $U \in \mathcal{U}$, we define $L_{U} \in B\left(l^{2}(\mathcal{U})\right)$ such that

$$
L_{U} \chi_{V}=f(U V) \chi_{\sigma(U V)}, \quad V \in \mathcal{U} .
$$

Then $L$ is a unitary representation of $\mathcal{U}$ onto $l^{2}(\mathcal{U})$ such that $L_{U} L_{V}=f(U V) L_{\sigma(U V)}$ and $L_{U}^{-1}=f\left(U^{-1}\right) L_{\sigma\left(U^{-1}\right)}$ for all $U, V \in \mathcal{U}$. In the group case, this is exactly the left regular representation for the group. Thus we also call $L$ the left regular representation for the group-like unitary system $\mathcal{U}$. It is obvoius that every subrepresentation of the left regular representation admits a normalized tight frame vector.

By using the polar decomposition for the analysis operator $T_{x}$ (usually unbounded) associated with a cyclic vector $x$ for a group-like unitary system $\mathcal{U}$, we are able to prove that $\mathcal{U}$ is unitarily equivalent to a subrepresentation of the regular representation for the group-like unitary system. Thus we have the following ([14]):

Theorem 3.1 Suppose that $x$ is a cyclic vector for a group-like unitary system $\mathcal{U}$ and $B_{\mathcal{U}}$ is dense in $H$. Then there is a vector $\eta \in H$ such that $\mathcal{U} \eta$ is a normalized tight frame for $H$.

In other words, if $\mathcal{U}$ is a group-like unitary system such that $B_{\mathcal{U}}$ is dense in $H$, then every cyclic invariant subspace of $\mathcal{U}$ admits a normalized tight frame vector for the subspace. The well-known density theorem for Gabor systems will follow from Theorem 3.1 and the following simple argument:

Corollary 3.2 [Density Theorem] If there is a function $g \in L^{2}\left(R^{d}\right)$ such that $\mathbf{G}(\mathcal{L}, \mathcal{K}, g)$ is complete in $L^{2}\left(R^{d}\right)$. Then $\mathrm{v}(\mathcal{L}) \mathrm{v}(\mathcal{K}) \leq 1$.

Proof. Write $\mathcal{L}=A Z^{d}$ and $\mathcal{K}=B Z^{d}$ with $A, B$ both $d \times d$ nonsingular real matrices. From Theorem 3.1, we can assume that $\mathbf{G}(\mathcal{L}, \mathcal{K}, g)$ is a normalized tight frame for $L^{2}\left(R^{d}\right)$. It is also possible (see [citenumGH1]) to find a sequence $\left\{f_{n}\right\} \subset L^{2}\left(R^{d}\right)$ such that

$$
\bigcup_{n} \mathbf{G}\left(\mathcal{L}, \mathcal{K}, f_{n}\right)
$$

is a normalized tight frame for $L^{2}\left(R^{d}\right)$ with the property that

$$
\sum_{n}\left\|f_{n}\right\|^{2}=|\operatorname{det}(A B)|
$$

Let $(f)_{\ell, \kappa}=e^{2 \pi i<\ell, x>} f(x-\kappa)$. Using the fact

$$
\left|<g,\left(f_{n}\right)_{\ell, \kappa}>\right|=\left|<(g)_{-\ell,-\kappa}, f_{n}>\right|,
$$


we have

$$
\begin{aligned}
1 & \geq\|g\|^{2}=\sum_{n} \sum_{\ell \in \mathcal{L}, \kappa \in \mathcal{K}}\left|<g,\left(f_{n}\right)_{\ell, \kappa}>\right|^{2} \\
& =\sum_{n} \sum_{\ell \in \mathcal{L}, \kappa \in \mathcal{K}}\left|<(g)_{-\ell,-\kappa}, f_{n}>\right|^{2} \\
& =\sum_{n}\left\|f_{n}\right\|^{2}=|\operatorname{det} A B| .
\end{aligned}
$$

Thus $\mathrm{v}(\mathcal{L}) \mathrm{v}(\mathcal{K}) \leq 1$, as expected.

From Corollary 3.2 and the lattice tiling result in [22], we have

Theorem 3.3 [22] Let $\mathcal{L}$ and $\mathcal{K}$ be two full-rank lattices in $R^{d}$. Then the following are equivalent:

(i) $\mathrm{v}(\mathcal{L}) \mathrm{v}(\mathcal{K}) \leq 1$.

(ii) There is $g \in L^{2}\left(R^{d}\right)$ such that $\mathbf{G}(\mathcal{L}, \mathcal{K}, g)$ is complete in $L^{2}\left(R^{d}\right)$.

(iii) There is $g \in L^{2}\left(R^{d}\right)$ such that $\mathbf{G}(\mathcal{L}, \mathcal{K}, g)$ is a frame for $L^{2}\left(R^{d}\right)$.

\section{THE UNIQUE DUAL PROPERTY}

In application, it is also important to know the properties of the dual frames since it is the dual that provides the discrete data in recovering signals by the reconstruction formula. In general a frame can have many duals. In fact a frame $\left\{x_{n}\right\}$ has a unique dual if and only if it is a Riesz basis (cf. [20]). However, if we work with special kind of frames, it is possible that there is only one dual of the same kind even if the frame is not a Riesz basis. We are interested in characterizing those frames, induced from a special class of unitary systems, that have unique duals of the same kind. In particular we are interested in the following question:

Question 3: Let $\mathcal{L}$ and $\mathcal{K}$ be two full-rank lattices in $R^{d}$ and let $g \in L^{2}\left(R^{d}\right)$ be such that it generates a Gabor frame for the closed subspace generated by $\mathbf{G}(\mathcal{L}, \mathcal{K}, g)$ (In this case, we say that $g$ generates a subspace Gabor frame). Under what conditions on $g$ do we have a unique function $h \in \overline{\operatorname{span}} \mathbf{G}(\mathcal{L}, \mathcal{K}, g)$ such that $\mathbf{G}(\mathcal{L}, \mathcal{K}, h)$ is a dual of $\mathbf{G}(\mathcal{L}, \mathcal{K}, g) ?$

We remark that similar question for wavelet frames has be discussed by C. K. Chui, X. Shi and J. Stockler [7]. The answer to the above question for a special case is known ( $\mathrm{cf}[2])$ because of the density result discussed in section 3: Suppose that $\mathbf{G}(\mathcal{L}, \mathcal{K}, g)$ is a Gabor frame for $L^{2}(R)$ with $\mathcal{L}=\alpha Z$ and $\mathcal{K}=\beta Z(\alpha, \beta>0)$. Then from the density theorem we have $\alpha \beta \leq 1$. If $\alpha \beta=1$, then $\mathbf{G}(\mathcal{L}, \mathcal{K}, g)$ must be a Riesz basis and so the dual is always unique. In the case that $\alpha \beta<1$ (so $1 / \alpha \beta>1$ ), again by the density theorem $\left\{e^{2 \pi i(1 / \beta) m} g(x-(1 / \alpha) n): m, n \in Z\right\}$ is not complete for $L^{2}(R)$. Hence we are able to pick up some non-zero function $h_{1}$ in the orthogonal complement of $\left\{e^{2 \pi i(1 / \beta) m} g(x-(1 / \alpha) n): m, n \in Z\right\}$ such that $\left.\mathbf{G}\left(\mathcal{L}, \mathcal{K}, h_{1}\right)\right)$ is a Bessel sequence (need some arguments). Assume that $h$ generates the standard dual for $\mathbf{G}(\mathcal{L}, \mathcal{K}, g)$. Then it can be shown that $\mathbf{G}\left(\mathcal{L}, \mathcal{K},\left(h+h_{1}\right)\right.$ is a dual for $\mathbf{G}(\mathcal{L}, \mathcal{K}, g)$. Clearly this dual is different from the standard dual since $h_{1} \neq 0$. Hence the dual is not unique. However, this argument does not apply to the subspace Gabor frame case and to the more general group-like unitary system situation.

To answer the above question, we can apply operator algebra techniques to obtain a characterization for those frame vectors (for a group-like unitary system) that have unique dual frame vectors. A special application of this will give us a complete solution for Question 3.

We first note that if two frame vectors $\eta$ and $\xi$ for a group-like unitary system $\mathcal{U}$ are similar (i.e., there is an invertible operator $S$ on $H$ such that $S U \eta=U \xi$ for all $U \in \mathcal{U}$ ), and one of them has a unique dual frame vector, then so does the other. Therefore it suffices for us to consider the normalized tight frame vectors since every frame vector for a group-like unitary system is similar to a normalized tight one. 
Here is the general characterization $([15])$ :

Theorem 4.1 Assume that $\eta$ is a complete normalized tight frame vector for a group-like unitary system $\mathcal{U}$. Then the following are equivalent:

(i) $\eta$ has a unique dual frame vector.

(ii) $\langle\eta, U V \eta\rangle=\langle\eta, V U \eta>$ holds for all $U, V \in \mathcal{U}$.

(iii) For every complete normalized tight frame vector $\xi$ of $\mathcal{U}$, there is a (unique) unitary operator $V$ in $\mathcal{U}^{\prime}$ such that $V \eta=\xi$.

(iv) For every complete frame vector $\xi$ of $\mathcal{U}$, there is a (unique) invertible operator $T$ in $\mathcal{U}^{\prime}$ such that $T \eta=\xi$.

(v) $\overline{\operatorname{span}}\left\{\mathcal{U}^{\prime} \eta\right\}=H$.

Condition (ii) in Theorem 4.1 is the most useful one that can be easily checked and applied to various concrete unitary systems. For example, the following is immediate from (ii):

Corollary 4.2 If $\mathcal{U}$ is an abelian group unitary system, then for each complete frame vector of $\mathcal{U}$ there is a unique dual frame vector.

Applying Theorem 4.1 to Gabor systems we have $\left(\mathcal{U}=\mathcal{U}_{\mathcal{L}, \mathcal{K}}, H=M\right)$ :

Theorem 4.3 Let $\mathbf{G}(\mathcal{L}, \mathcal{K}, g)$ is a normalized tight frame for $M$, the closure of it linear span. Then $g$ has a unique Gabor dual in $M$ if and only if

$$
e^{2 \pi i<\ell, \kappa^{\prime}>}<g, g_{\ell, \kappa}>=e^{2 \pi i<\ell^{\prime}, \kappa>}<g, g_{\ell, \kappa}>
$$

holds for all $\ell, \ell^{\prime} \in \mathcal{L}$ and $\kappa, \kappa^{\prime} \in \mathcal{K}$, where $g_{\ell, \kappa}(x)=e^{2 \pi<\ell, x>} g(x-\kappa)$.

For the one-dimensional $(d=1)$ case, we can say more. In what follows we always assume that $\mathcal{L}=\alpha Z$ and $\mathcal{K}=\beta Z(\alpha, \beta>0)$.

Corollary 4.4 Suppose that g generates a normalized tight subspace Gabor frame for the closure (say, $M$ ) of it linear span. Then $\mathbf{G}(\mathcal{L}, \mathcal{K}, g)$ has a unique Gabor dual in $M$ if and only if the identity

$$
e^{2 \pi i \alpha \beta(n k-m l)}<g,(g)_{m \alpha, n \beta}>=<g,(g)_{m \alpha, n \beta}>
$$

holds for all $m, n, k, \ell \in Z$, where $(g)_{m \alpha, n \beta}=e^{2 \pi i m \alpha x} g(x-\beta n)$.

Corollary 4.4 will immediately implies that $\mathbf{G}(\mathcal{L}, \mathcal{K}, g)$ has a unique Gabor dual if $\alpha \beta$ is an integer, and when $\alpha \beta$ is irrational, then $\mathbf{G}(\mathcal{L}, \mathcal{K}, g)$ has a unique Gabor dual if and only if $\mathbf{G}(\mathcal{L}, \mathcal{K}, g)$ is a Riesz sequence. However it is much more complicated to deal with the rational (but not integer) case. By Corollary 4.4 and a short argument, we can get:

Proposition 4.5 Let $\alpha \beta=\frac{p}{q}$ with $\operatorname{gcd}(p, q)=1$ and let $\mathbf{G}(\mathcal{L}, \mathcal{K}, g)$ be a normalized tight Gabor frame for $M$, the closure of it linear span. Then the following are equivalent:

(i) $\mathbf{G}(\mathcal{L}, \mathcal{K}, g)$ has a unique Gabor dual in $M$

(ii) $<g, g_{m \alpha, n \beta}>=0$ for all $(m, n) \notin q Z \times q Z$.

(iii)

$$
\sum_{l \in Z} g\left(x-\frac{l}{\alpha} \overline{g\left(x-\frac{l+p k}{\alpha}-\frac{p j}{\alpha q}\right)}=0, \quad k \in Z, \quad 1 \leq j \leq q-1\right.
$$


and

$$
\sum_{l \in Z} g\left(x-\frac{l}{\alpha q}\right) \overline{g\left(x-\frac{l}{\alpha q}-\frac{p k}{\alpha q}\right)} e^{2 \pi i \frac{j l}{q}}=0, \quad k \in Z, 1 \leq j \leq q-1
$$

Recall that the Zak transform of a function $f \in L^{2}(R)$ is

$$
(\mathcal{Z} f)(t, \nu)=\sum_{k \in Z} f(t-k) e^{2 \pi i k \nu}, \quad \text { a. e. } \quad t, \nu \in R
$$

where the right-hand side has to be interpreted in $L_{l o c}^{2}\left(R^{2}\right)$-sense. Zak transform is completely determined by its values in the unit square $Q=[0,1) \times[0,1)$. Moreover it is a unitary map of $L^{2}(R)$ onto $L^{2}(Q)$. To deal with the rational case for our problem, we need to introduce the vector-valued Zak transform: Let $q$ be a positive integer.

The vector-valued Zak transform of a function $f \in L^{2}(R)$ with respect to $q$ is

$$
\left(\mathcal{Z}_{q} f\right)(t, \nu)=\left(\begin{array}{c}
(\mathcal{Z} f)(t, \nu) \\
(\mathcal{Z} f)\left(t+\frac{1}{q}, \nu\right) \\
\cdot \\
\cdot \\
\cdot \\
(\mathcal{Z} f)\left(t+\frac{q-1}{q}, \nu\right)
\end{array}\right)=\left(\begin{array}{c}
\sum_{k \in Z} f(t-k) e^{2 \pi i k \nu} \\
\sum_{k \in Z} f\left(t+\frac{1}{q}-k\right) e^{2 \pi i k \nu} \\
\cdot \\
\cdot \\
\cdot \\
\sum_{k \in Z} f\left(t+\frac{\dot{q}-1}{q}-k\right) e^{2 \pi i k \nu}
\end{array}\right)
$$

Since $\mathcal{Z}$ is a unitary from $L^{2}(R)$ onto $L^{2}\left([0,1]^{2}\right)$, it follows that $\mathcal{Z}_{q}$ is a unitary transform from $L^{2}(R)$ onto the super-space $L^{2}\left(\left[0, \frac{1}{q}\right] \times[0,1]\right) \oplus \ldots \oplus L^{2}\left(\left[0, \frac{1}{q}\right] \times[0,1]\right)$.

Using Proposition 4.5 and the vector-valued Zak transform we are able to prove the following ([15]):

Theorem 4.6 Let $\mathbf{G}(\mathcal{L}, \mathcal{K}, g)$ be a frame for the closure (say, $M$ ) of its linear span and $g \neq 0$.

(i) If $\alpha \beta<1, \mathbf{G}(\mathcal{L}, \mathcal{K}, g)$ has more than one Gabor duals in $M$

(ii) If $\alpha \beta=k \in N$, then $\mathbf{G}(\mathcal{L}, \mathcal{K}, g)$ has a unique Gabor dual in $M$.

(iii) If $\alpha \beta$ is irrational, then $\mathbf{G}(\mathcal{L}, \mathcal{K}, g)$ has a unique Gabor dual in $M$ if and only if $\mathbf{G}(\mathcal{L}, \mathcal{K}, g)$ is a Riesz sequence.

The case where $\alpha \beta>1$ is rational but not an integer remains open.

\section{REFERENCES}

1. J. Benedetto and D. Walnut, "Gabor frames for $L^{2}$ and related spaces," Wavelets: Mathematics and Applications, Eds. J. Benedetto and M. Frazier, CRC Press Inc., 1994.

2. P. Casazza, "Modern tools for Weyl-Heisenberg (Gabor) frame theory," preprint.

3. P. Casazza and O. Christensen, "Weyl-Heisenberg frames for subspaces in $L^{2}(R)$," Proc. Amer. Math. Soc., to appear.

4. P. Casazza and O. Christensen, "Classifying tight Weyl-Heisenberg frames," preprint.

5. P. Casazza, D. Han and D. Larson, "Frames for Banach spaces," Contemp. Math., 247, pp. 149-182, 1999.

6. O. Christensen, B. Deng and C. Heil, "Density of Gabor frames," Appl. Comput. Harm. Anal, 7, pp. 292-304, 1999

7. C. K. Chui, X. Shi and J. Stockler, "Affine frames, quasi-affine frames, and their duals," Adv. Comput. Math., 8, pp. 1-17, 1998.

8. X. Dai and D. Larson, "Wandering vectors for unitary systems and orthogonal wavelets," Memoirs Amer. Math. Soc., 640, 1998. 
9. I. Daubechies, "The wavelet transformation, time-frequence localization and signal analysis," IEEE Trans. Inform. Theory, $\mathbf{3 6}$, pp. 961-1005, 1990.

10. I. Daubechies, Ten Lectures on Wavelets, CBMS-NSF regional conference series in Applied Math., Philadelphia, 1992.

11. I. Daubechies, H. Landau and Z. Landau, "Gabor time frequency lattices and the Wexler-Raz identity" J. Fourier. Anal. Appl., 1, pp. 437 -478, 1995.

12. H.G.Feichtinger and T.Strohmer (eds), Gabor Analysis and Algorithms: Theory and Applications, Applied and Numerical Harmonic Analysis, Birkhuser, 1998.

13. J. P. Gabardo and D. Han, "Frame representations for group-like unitary operator systems," preprint.

14. J. P. Gabardo and D. Han, "Subspace Weyl-Heisenberg frames," preprint.

15. J. P. Gabardo and D. Han, "Weyl-Heisenberg dual frames and operator algebras," preprint.

16. D. Gabor, "Theory of Communication," J. Inst. Elec. Eng., (London), 93, pp. 429-457, 1946.

17. K. Grochenig, "Gabor analysis and LCA groups," Gabor Analysis and Algorithms: Theory and Applications, Applied and Numerical Harmonic Analysis, (eds. H.G.Feichtinger and T.Strohmer) Birkhuser, pp. 211-231, 1998.

18. D. Han, "Wandering vectors for irrational unitary systems," Trans. Amer. Math. Soci, 350, pp. 321-329, 1998.

19. D. Han, "Unitary systems, wavelets and operator algebras," Texas A\&M University, Ph.D. Thesis, 1998.

20. D. Han and D. Larson, "Frames, bases and group representations," Memoirs Amer. Math. Soc., to appear,

21. D. Han, D. Larson, M. Papadakis and T. Stavropoulos, "Multiresolution analysis of abstract Hilbert spaces and wandering subspaces," Contemp. Math., 247, pp. 259 - 284, 1999.

22. D. Han and Y. Wang, "Lattice tiling and the Weyl-Heisenberg frames," preprint.

23. A. J. E. Janssen, "Signal analytic proofs of two basic results on lattice expansions," Appl. Comp. Harm. Anal. 1, pp. 350-354, 1994.

24. D. Larson, "Von Neumann algebras and wavelets. Operator algebras and applications" (Samos, 1996), NATO Adv. Sci. Inst. Ser. C Math. Phys. Sci., 495, pp. 267-312, Kluwer Acad. Publ., Dordrecht, 1997

25. D. Larson, "Frames and wavelets from an operator-theoretic point of view. Operator algebras and operator theory" (Shanghai, 1997), Contemp. Math., 228, pp. 201-218, 1998.

26. M. A. Rieffel, "Von Neumann algebras associated with pairs of lattices in Lie groups" Math. Ann., 257, pp. 403-413, 1981.

27. J. Ramanathan and T. Steger, "Incompleteness of sparse coherent states," Appl. Comp. Harm. Anal., 2, pp. 148-153, 1995.

28. A. Ron and Z. Shen, "Weyl-Heisenberg frames and Riesz bases in $L^{2}\left(R^{d}\right)$," Duke Mathematical Journal, 89, pp. 237-282, 1997.

29. J. von Neumann, Mathematical Foundations of Quantum Mechanics, Princeton University Press, Princeton, NJ. 1932, 1945, 1955. 